\title{
Méningite à Mycobacterium tuberculosis chez une immigrante récemment arrivée au Canada
}

\author{
Adam S. Komorowski MD, Carson K.L. Lo MD, Neal Irfan DPharm, Nishma Singhal MD
}

Citation : CMAJ 2021 November 29;193:E1807-10. doi : 10.1503/cmaj.210740-f

Voir la version anglaise de l'article ici : www.cmaj.ca/lookup/doi/10.1503/cmaj.210740

$\mathbf{U}$ ne femme de 37 ans a consulté dans un hôpital communautaire pour céphalée frontale intense, fièvre subjective et épisodes confusionnels depuis 1 semaine. Elle a déclaré n'avoir eu ni nausées, ni vomissements, ni convulsions ou érythème cutané. Elle était arrivée comme immigrante au Canada 8 mois auparavant en provenance du Nigéria, n'avait pas voyagé depuis, et n'avait eu aucun contact avec des personnes ou des animaux malades.

À l'admission, la patiente était fiévreuse $\left(38,3^{\circ} \mathrm{C}\right)$, mais ses autres signes vitaux étaient sans particularités. Elle présentait une rigidité de la nuque sans déficits neurologiques focaux. Ses analyses sanguines ont montré une numération leucocytaire normale et une numération absolue des lymphocytes à 0,7 (plage normale $1,0-3,57) \times 10^{9}$ cellules/L. Les tests de dépistage du VIH étaient négatifs, tout comme les frottis sanguins pour détection des parasites de la malaria. Un premier examen du liquide céphalorachidien (LCR) a montré une numération leucocytaire à 96 (plage normale $0-5) \times 10^{6}$ cellules/L ( $59 \%$ de lymphocytes, $36 \%$ de neutrophiles et $2 \%$ de monocytes) et des taux de glucose à 1,9 (plage normale 2,2-3,9) $\mathrm{mmol} / \mathrm{L}$ et de protéines à 1,20 (normale $<0,45) \mathrm{g} / \mathrm{L}$. À l'admission, son taux d'aspartate aminotransférase était à 13 (plage normale 10-40) U/L et son taux d'alanine aminotransférase était à 18 (plage normale 5-42) U/L. Les résultats des cultures bactériennes et fongiques du LCR, du test PCR pour le virus de l'herpès simplex et les entérovirus, du test à flux latéral pour détecter l'antigène cryptococcique et des analyses pour l'encéphalite autoimmune étaient tous négatifs. On n'a pas demandé de culture mycobactérienne du LCR.

La crainte d'une méningo-encéphalite a justifié le recours à une tomodensitométrie (TDM) cérébrale sans agent de contraste qui a révélé une petite zone d'hypodensité sous-corticale au lobe temporal droit, évocatrice d'une encéphalite. Ses médecins ont commencé un traitement empirique par ceftriaxone, vancomycine et acyclovir. Au jour 5, la patiente a cessé de parler et est devenue confuse; elle a développé une paralysie du muscle droit latéral de l'œil gauche, avec pupille ipsilatérale aréactive à $6 \mathrm{~mm}$. L'imagerie par résonance magnétique du cerveau a montré une intensité de signal élevée dans l'ensemble des sillons de la convexité et des citernes basales qui est compatible avec une méningite (figure 1). Une TDM du thorax, de l'abdomen et du bassin a montré une lymphadénopathie abdominale non spécifique. La

\section{Points clés}

- Au Canada, la méningite tuberculeuse est une rare infection à Mycobacterium tuberculosis, qui s'observe davantage chez des personnes immigrantes au cours des 5 années qui suivent leur arrivée au Canada.

- Chez les patients qui ont des facteurs de risque à l'égard de la tuberculose (TB), un diagnostic présumé de méningite tuberculeuse devrait être envisagé si le tableau clinique et les résultats des analyses du liquide céphalorachidien concordent; un traitement empirique doit être amorcé sans tarder.

- Les médecins doivent exercer une surveillance étroite de toute réaction indésirable aux antituberculeux, comme la transaminite et l'insuffisance rénale aiguë, et cesser tout médicament potentiellement hépatotoxique, si possible.

- Les patients qui présentent une hydrocéphalie chronique comme complication de leur méningite pourraient avoir besoin d'une corticothérapie prolongée en plus d'un drainage extraventriculaire.

patiente a développé un réflexe de Cushing et elle a été intubée avant d'être transférée dans notre établissement de soins tertiaires pour une intervention neurochirurgicale. Les neurochirurgiens ont inséré un drain ventriculaire externe pour soulager l'hydrocéphalie et ont intensifié l'antibiothérapie avec méropénem, vancomycine et acyclovir.

Nous avons consulté l'équipe d'infectiologie selon laquelle la méningite à Mycobacterium tuberculosis était le principal diagnostic différentiel, compte tenu des facteurs de risque de la patiente, de l'évolution de son tableau clinique en dépit des antimicrobiens administrés, des résultats des analyses biochimiques du LCR et de la lymphadénopathie abdominale. Nous avons cessé l'antibiothérapie, effectué une autre ponction lombaire en vue d'une culture mycobactérienne au moyen du test d'amplification des acides nucléiques GeneXpert MTB/RIF (TAAN; Cepheid) et débuté un traitement empirique pour la méningite tuberculeuse (TB) par dexaméthasone intraveineuse (IV) $0,4 \mathrm{mg} / \mathrm{kg} / \mathrm{j}$ administrée toutes les 4 heures, rifampine IV $10 \mathrm{mg} / \mathrm{kg} / \mathrm{j}$, et par voie orale, isoniazide $5 \mathrm{mg} / \mathrm{kg} / \mathrm{j}$, pyrazinamide $25 \mathrm{mg} / \mathrm{kg} / \mathrm{j}$ et éthambutol $15 \mathrm{mg} / \mathrm{kg} / \mathrm{j}$. Nous n'avons observé aucun bacille acidorésistant à la coloration du LCR et le TAAN s'est révélé 


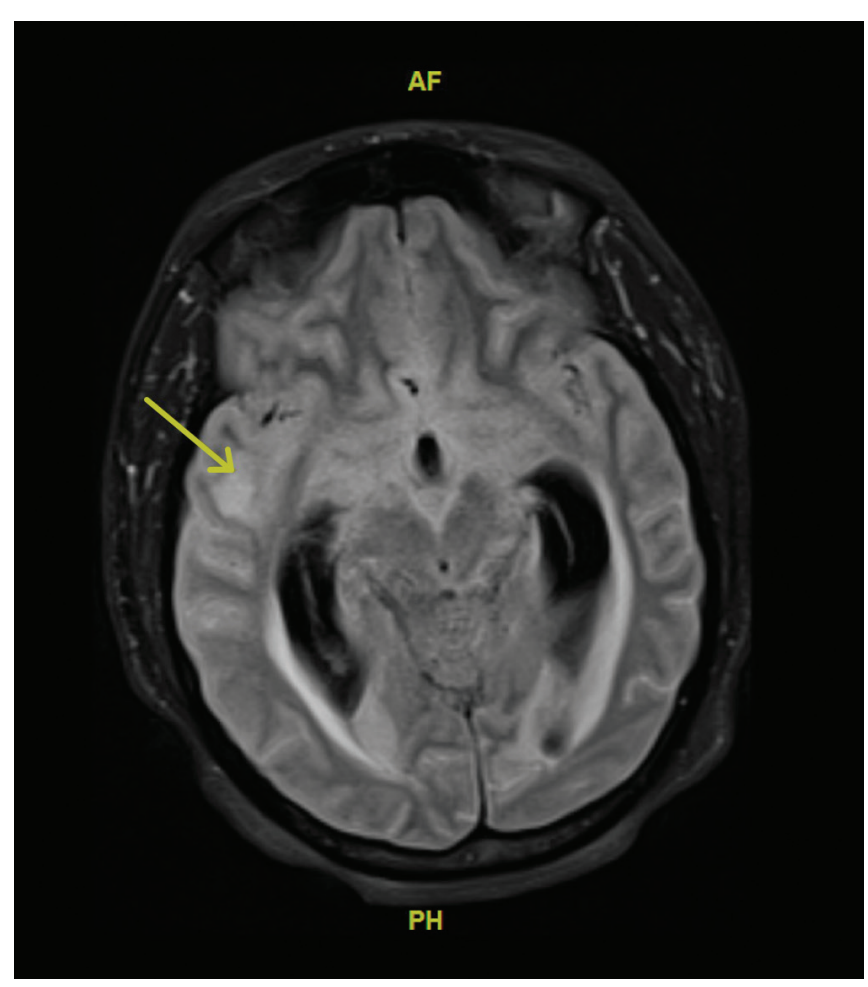

Figure $1:$ Imagerie par résonance magnétique (IRM) du cerveau d'une femme de 37 ans atteinte de méningite tuberculeuse obtenue au jour 5 de son hospitalisation. Cette image axiale FLAIR (fluid-attenuated inversion recovery) au niveau des citernes basales et des lobes temporaux montre une intensité de signal élevée dans l'ensemble des sillons de la convexité et des citernes basales qui est compatible avec une méningite. Les 2 cornes temporales des ventricules latéraux sont dilatées, avec intensité de signal anormale tout autour, ce qui est compatible avec une hydrocéphalie et un passage transépendymaire de liquide céphalorachidien. Le rapport initial d'IRM présentait une éventuelle anomalie parenchymateuse dans le lobe temporal droit (flèche jaune), présumée compatible avec une encéphalite. Cependant, après examen de cette image, les résultats de l'IRM se révèlent compatibles surtout avec une méningite.

négatif à l'égard du complexe $M$. tuberculosis. Nos intensivistes ont effectué une bronchoscopie, et le TAAN, la coloration et la culture sur les spécimens prélevés ont tous été négatifs.

À partir du jour 18, des effets indésirables ont nécessité une série de changements au schéma thérapeutique de la patiente (figure 2). Ses taux d'aspartate aminotransférase (141 U/L) et d'alanine aminotransférase (227 U/L) se sont élevés à plus de 5 fois la limite supérieure de la normale, ce qui nous a forcés à cesser le pyrazinamide et l'isoniazide. Nous les avons remplacés par de l'amikacine IV $15 \mathrm{mg} / \mathrm{kg} / \mathrm{j}$ et de la lévofloxacine IV $10 \mathrm{mg} / \mathrm{kg} / \mathrm{j}$. L'amikacine a causé une insuffisance rénale aiguë et a multiplié par 2 le taux de créatinine sérique en l'espace de 4 jours; nous sommes donc passés à du linézolide par voie orale $600 \mathrm{mg} 2$ fois par jour. Une fois les taux de transaminase revenus à la normale, nous avons cessé le linézolide et recommencé l'isoniazide. Comme la patiente a ensuite développé une transaminite que nous avons attribuée à l'isoniazide, nous avons cessé définitivement l'isoniazide et recommencé le linézolide.

M. tuberculosis a été détecté dans la culture du LCR au jour 37. L'isolat était négatif pour ce qui est des mutations causant une résistance à la rifampine et à l'isoniazide et plus tard, le laboratoire a confirmé la sensibilité aux agents de première intention (c.-à-d., isoniazide, rifampine, pyrazinamide, éthambutol) et de deuxième intention (c.-à-d., éthionamide, linézolide, moxifloxacine).

Nos premières tentatives de sevrer la patiente de la dexaméthasone conformément aux schémas habituels ont été infructueuses en raison d'une hydrocéphalie persistante; nous avons donc maintenu le traitement par dexaméthasone durant ses hospitalisations et nous l'avons fait passer à de l'hydrocortisone au moment du congé pour permettre un sevrage graduel de la corticothérapie sur une période de 5 mois (figure 3 ). Après une période de sevrage, le drain ventriculaire externe a été retiré au jour 31. La patiente a été extubée au jour 35; peu après, toutefois, les neurochirurgiens ont posé un autre drain ventriculaire externe en raison d'une aggravation de l'hydrocéphalie; ils l'ont éventuellement changé pour une dérivation ventriculo-péritonéale (DVP) programmable qui a ensuite été remplacée par une DVP munie d'une valve à pression moyenne après la survenue d'une brève aggravation de l'hydrocéphalie et de myoclonies généralisées associées à un risque de convulsions. La patiente a fini par recevoir son congé de l'hôpital communautaire au jour 148.

La patiente était stable lors du suivi 1 an plus tard et ses antituberculeux ont été cessés. Même si elle a retrouvé son autonomie fonctionnelle et son élocution, elle présente des séquelles persistantes de la maladie et des complications du traitement, particulièrement un syndrome de perte de sel d'origine cérébrale et une atteinte bilatérale des nerfs optiques; la patiente n'a pas pu retourner au travail.

\section{Discussion}

La tuberculose demeure une importante cause de décès à l'échelle mondiale, avec 1,4 million de victimes en $2019^{1}$. Au Canada, la majorité des cas déclarés de TB s'observe chez des personnes nées à l'étranger et plus de $40 \%$ s'observent au cours des 5 années suivant leur arrivée ${ }^{1}$. La tuberculose du système nerveux central représente $1 \%-2 \%$ de tous les cas de TB et provoque une maladie qui peut se manifester par abcès cérébral, cérébrite, méningite, myélite et tuberculomes. La méningite tuberculeuse en est la manifestation la plus fréquente au niveau du système nerveux central, avec des taux de morbidité $(25 \%)$ et de mortalité (15\%-40\%) substantiels, malgré un traitement approprié2. Les facteurs de risque indépendants de décès chez les patients hospitalisés pour méningite tuberculeuse incluent infection au VIH, âge de plus de 40 ans, culture de LCR positive à l'égard de la TB et gravité du tableau clinique ${ }^{3}$.

La méningite tuberculeuse s'accompagne en général de symptômes subaigus tels que fièvre, céphalées, malaise et changements de personnalité, et peut évoluer vers une paralysie des nerfs crâniens et autres déficits neurologiques focaux, des convulsions, le coma et le décès. La ponction lombaire est essentielle puisque les analyses du LCR peuvent faciliter le diagnostic de méningite tuberculeuse et guider l'amorce d'un traitement empirique. Les signes typiques aux analyses du LCR incluent pression d'ouverture élevée, pléocytose lymphocytaire marquée, 


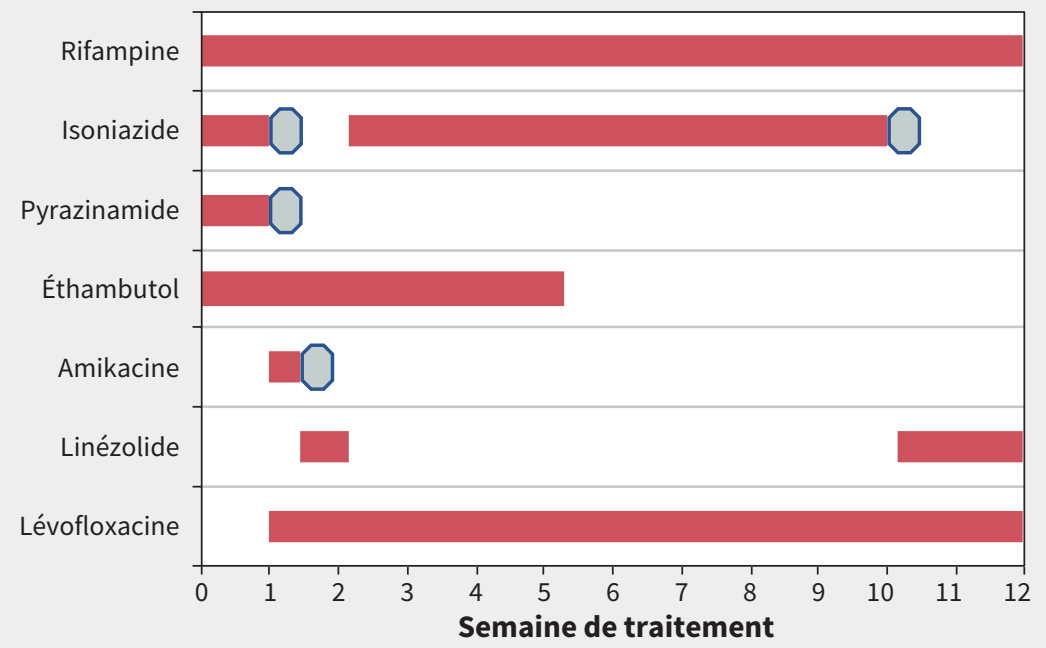

Figure 2 : Représentation schématique des changements apportés au traitement pour méningite tuberculeuse au cours des 3 premiers mois suivant le diagnostic; les effets indésirables des antimicrobiens sont représentés par des octogones. Une élévation asymptomatique des aminotransférases à plus de 5 fois la limite supérieure de la normale a forcé l'arrêt de l'isoniazide et du pyrazinamide. Nous avons ajouté de l'amikacine, mais l'avons cessée après 3 jours en raison d'une insuffisance rénale aiguë. Nous avons utilisé le linézolide comme traitement provisoire jusqu'à ce que les taux d'aminotransférase reviennent à la normale; nous avons ensuite recommencé l'isoniazide. Nous avons éventuellement cessé l'éthambutol parce que la souche de Mycobacterium tuberculosis de la patiente s'est révélée sensible à la rifampine et à l'isoniazide. À la semaine 10 du traitement, la patiente a présenté une hépatite qui nous a forcés à cesser définitivement l'isoniazide et à réinstaurer le linézolide.

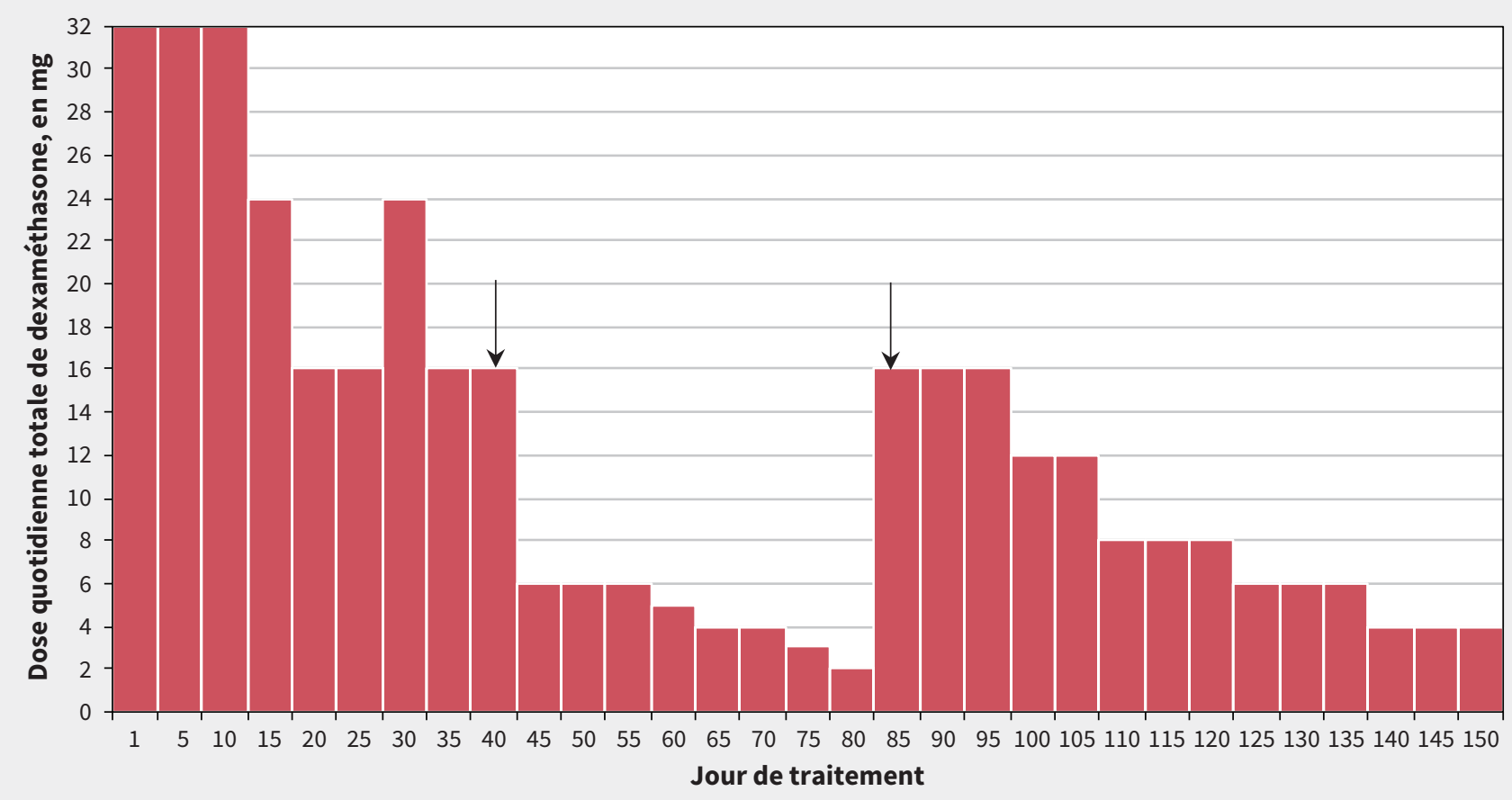

Figure 3 : Dose quotidienne totale de dexaméthasone durant l'hospitalisation de la patiente pour méningite tuberculeuse. Du jour 1 au jour 40 , la patiente a été traitée dans notre centre de soins tertiaires; la flèche au jour 40 indique son retour vers un autre hôpital où elle est restée jusqu'au jour 85. La flèche vis-à-vis du jour 85 indique sa réadmission dans notre centre de soins tertiaires pour une aggravation de son hydrocéphalie. Elle a été transférée de nouveau à l'autre hôpital au jour 87; elle est y est restée jusqu'au moment de son congé au jour 148. Au moment de son congé, on a remis à la patiente une ordonnance d'hydrocortisone qui a été sevrée graduellement sur une période de 5 mois. 
taux élevé de protéines dû à l'obstruction de la circulation du LCR et baisse du glucose.

Les analyses microbiologiques permettent de confirmer le diagnostic de méningite tuberculeuse, mais comportent des limites. Les spécimens de LCR doivent être envoyés pour coloration et examen au microscope des bacilles acidorésistants, TAAN et culture. Toutefois, le fardeau mycobactérien dans le LCR est généralement moindre chez les patients atteints de méningite tuberculeuse que chez ceux qui font une TB pulmonaire, ce qui affecte le rendement des épreuves microbiologiques. La sensibilité de la coloration et de la microscopie pour le dépistage des bacilles acidorésistants (20\%-80\%) est plus faible chez les patients qui ont une TB extrapulmonaire et chez les porteurs du $\mathrm{VIH}$; un résultat négatif à la coloration des bacilles acidorésistants du LCR ne permet donc pas aux médecins d'écarter le diagnostic de méningite tuberculeuse ${ }^{1}$. Toutefois, on peut augmenter la sensibilité de la coloration pour dépistage des bacilles acidorésistants dans la méningite tuberculeuse en effectuant des ponctions lombaires quotidiennes pendant 3 jours, en soumettant le dernier tube prélevé et en recueillant un fort volume $(10-15 \mathrm{~mL})^{1}$. De même, s'il est positif, le TAAN a l'avantage d'offrir un diagnostic présumé en moins de 24 heures, mais il est moins sensible avec le LCR qu'avec les expectorations ${ }^{2,4}$. Une TB pulmonaire s'observe concomitamment chez $22 \%-24 \%$ des patients atteints de méningite tuberculeuse et l'examen des expectorations est une modalité diagnostique moins effractive; les patients chez qui on soupçonne une méningite tuberculeuse devraient donc faire l'objet d'un dépistage de la TB pulmonaire ${ }^{1}$. Dans notre cas, ces tests se sont révélés négatifs.

Il faut parfois jusqu'à 7 semaines pour obtenir les résultats des cultures mycobactériennes et un résultat négatif ne permet pas toujours d'écarter le diagnostic de méningite tuberculeuse chez les patients qui n'ont pas d'autres diagnostics et dont l'état s'est amélioré avec le traitement antituberculeux. Toutefois, si le tableau clinique et les résultats d'analyses du LCR concordent, un diagnostic présumé de méningite tuberculeuse doit être envisagé chez les patients qui ont des facteurs de risque à l'égard de la TB. Il est impératif d'instaurer un traitement empirique sans tarder, puisque le délai d'amorce du traitement antituberculeux approprié avant la survenue d'un coma est le plus solide prédicteur de la survie ${ }^{5}$.

Les lignes directrices actuelles recommandent 2 mois de traitement intensif avec rifampine, isoniazide, pyrazinamide et éthambutol pour une TB qui répond aux médicaments, suivi d'un traitement d'entretien avec rifampine et isoniazide pendant 10 mois de plus ${ }^{1,4,6}$. Aucune différence n'a été observée sur le plan de l'issue clinique ou des rechutes avec un traitement d'une durée de 6 mois $^{7}$. La rifampine et l'éthambutol demeurent les pierres angulaires du traitement intensif pour la méningite tuberculeuse parce que les petits essais qui ont été réalisés n'ont montré aucune amélioration des taux de mortalité lorsqu'on ajoutait des agents capables de pénétrer plus efficacement le LCR ${ }^{8}$.

Comme on le voit ici, le traitement antituberculeux requiert une surveillance étroite des effets indésirables. Le cas échéant, il faut identifier l'agent causal le plus probable, prendre la décision de le cesser temporairement ou définitivement et choisir une solution de rechange acceptable. Les médecins doivent envisager des agents de deuxième intention ayant des paramètres pharmacocinétiques acceptables au regard du LCR, comme la lévofloxacine, le linézolide, la moxifloxacine, la cyclosérine ou l'éthionamide. Ces paramètres nous ont orientés dans notre choix du linézolide 2 fois par jour durant la phase critique de la maladie, alors que les résultats des tests de sensibilité n'étaient pas encore disponibles, par opposition à la posologie uniquotidienne généralement utilisée lors des essais cliniques sur la méningite tuberculeuse. Une étude ${ }^{9}$ a montré une amélioration des paramètres biochimiques au niveau du LCR et du score à l'échelle de Glasgow avec le schéma que nous avons utilisé.

Les médicaments potentiellement hépatotoxiques doivent être cessés en présence d'une grave transaminite ${ }^{10}$. Les lignes directrices recommandent l'arrêt de la pyrazinamide, de l'isoniazide et de la rifampine, et leur remplacement par une fluoroquinolone et un agent injectable (p. ex., aminosides, capréomycine), ou une fluoroquinolone et un agent oral de deuxième intention (p. ex., linézolide) ${ }^{1}$. Nous avons suspendu les 2 agents les plus probablement à l'origine de la transaminite, l'isoniazide et le pyrazinamide, mais nous avons maintenu la rifampine pour éviter d'arrêter la majorité des agents de première intention pendant la phase critique de la maladie chez notre patiente.

Réussir le traitement de la méningite tuberculeuse requiert une approche multimodale pour gérer l'augmentation de la pression intracrânienne, incluant des corticostéroïdes qui réduisent le risque de mortalité ${ }^{5,11}$. La dose et la durée optimales de la corticothérapie restent à préciser; les lignes directrices suggèrent un sevrage en $6-8$ semaines ${ }^{1,4,6}$. Notre cas rappelle les limites de ces recommandations et l'importance d'adapter le traitement à chaque individu, car plusieurs tentatives de sevrage de la corticothérapie ont échoué chez notre patiente; il a ultimement fallu l'échelonner sur 48 semaines.

La gestion de l'hydrocéphalie associée, surtout si elle est non communiquante ${ }^{12}$, pourrait nécessiter une intervention neurochirurgicale, par exemple, la pose d'un drain ventriculaire externe à courte échéance et, comme pour notre patiente, une DVP pour une prise en charge définitive ${ }^{11}$. Les complications affectant les nerfs optiques sont responsables de la plupart des pertes visuelles associées à la méningite tuberculeuse et peuvent avoir plusieurs causes, mais elles ont été attribuées à une hydrocéphalie chronique chez notre patiente en raison du moment de son déclenchement ${ }^{13}$. L'hyponatrémie peut être causée par un syndrome de perte de sel d'origine cérébrale ou un syndrome de sécrétion inappropriée de l'hormone antidiurétique, qu'il faut distinguer l'un de l'autre parce qu'on ne les traite différemment ${ }^{12}$. L'hyponatrémie de notre patiente a été attribuée à un syndrome de perte de sel d'origine cérébrale et corrigée au moyen de suppléments oraux de chlorure de sodium.

Notre cas illustre les étapes du diagnostic et la complexité de la prise en charge de la méningite tuberculeuse. La reconnaissance hâtive et l'instauration d'un traitement approprié, incluant une surveillance continue tout au long du décours de la maladie, maximisent la probabilité d'une récupération fonctionnelle. 


\section{Références}

1. Canadian tuberculosis standards 7th edition: 2014. Ottawa: Public Health Agency of Canada; 2014. Accessible ici : https://www.canada.ca/en/public -health/services/infectious-diseases/canadian-tuberculosis-standards-7th -edition.html (consulté le 17 avril 2021).

2. Thwaites GE, Tran TH. Tuberculous meningitis: many questions, too few answers. Lancet Neurol 2005;4:160-70.

3. Soria J, Metcalf T, Mori N, et al. Mortality in hospitalized patients with tuberculous meningitis. BMC Infect Dis 2019;19:9.

4. Guidelines for treatment of drug-susceptible tuberculosis and patient care, 2017 update. Geneva: World Health Organization; 2017. Accessible ici : https:// apps.who.int/iris/handle/10665/255052 (consulté le 17 avril 2021).

5. Prasad K, Singh MB, Ryan H. Corticosteroids for managing tuberculous meningitis. Cochrane Database Syst Rev 2016;(4):CD002244.

6. Nahid P, Dorman SE, Alipanah N, et al. Official American Thoracic Society/ Centers for Disease Control and Prevention/Infectious Diseases Society of America Clinical practice guidelines: treatment of drug-susceptible tuberculosis. Clin Infect Dis 2016;63:e147-95.

7. Jullien S, Ryan H, Modi M, et al. Six months therapy for tuberculous meningitis. Cochrane Database Syst Rev 2016;(9):CD012091.

8. Rizvi I, Malhotra HS, Garg RK, et al. Fluoroquinolones in the management of tuberculous meningitis: systematic review and meta-analysis. J Infect 2018;77:261-75.

9. Sun F, Ruan Q, Wang J, et al. Linezolid manifests a rapid and dramatic therapeutic effect for patients with life-threatening tuberculous meningitis. Antimicrob Agents Chemother 2014;58:6297-301.

10. Saukkonen JJ, Cohn DL, Jasmer RM, et al. An official ATS statement: hepatotoxicity of antituberculosis therapy. Am J Respir Crit Care Med 2006;174:935-52.

11. Davis A, Meintjes G, Wilkinson RJ. Treatment of tuberculous meningitis and its complications in adults. Curr Treat Options Neurol 2018;20:5. doi: 10.1007/ s11940-018-0490-9.

12. Donovan J, Figaji A, Imran D, et al. The neurocritical care of tuberculous meningitis. Lancet Neurol 2019;18:771-83.

13. Garg RK, Malhotra HS, Kumar N, et al. Vision loss in tuberculous meningitis. J Neurol Sci 2017;375:27-34.

\section{Intérêts concurrents : Aucun déclaré.}

Cet article a été révisé par des pairs.

Les auteurs ont obtenu le consentement de la patiente.

Affiliations : Division de microbiologie médicale (Komorowski), Département de pathologie et de médecine moléculaire; Programme des cliniciens-chercheurs (Komorowski); Division d'infectiologie (Lo, Irfan, Singhal), Département de médecine, Université McMaster, Hamilton, Ont.

Collaborateurs : Adam Komorowski était responsable de l'élaboration; Adam Komorowski et Carson Lo ont contribué à la méthodologie; Neal Irfan et Nishma Singhal étaient responsables de la supervision. Adam Komorowski et Carson Lo ont rédigé l'ébauche originale. Tous les auteurs ont révisé de façon critique le contenu intellectuel important du manuscrit; ils ont donné leur approbation finale pour la version destinée à être publiée et assument l'entière responsabilité de tous les aspects du travail.

Propriété intellectuelle du contenu : Il s'agit d'un article en libre accès distribué conformément aux modalités de la licence Creative Commons Attributions (CC BY-NC-ND 4.0), qui permet l'utilisation, la diffusion et la reproduction dans tout médium à la condition que la publication originale soit adéquatement citée, que l'utilisation se fasse à des fins non commerciales (c.-à-d., recherche ou éducation) et qu'aucune modification ni adaptation n'y soit apportée. Voir : https://creativecommons.org/licenses/by-nc-nd/4.0/deed.fr.

Remerciements : Les auteurs remercient la patiente d'avoir autorisé la publication de ce rapport de cas. Ils remercient en outre les $D^{\text {rs }}$ Sarah Brode, Ian Kitai, Julianne Kus et Anjali Shroff pour leur expertise et leurs précieux conseils initiaux relativement au traitement de cette patiente, ainsi que le $D^{r}$ Ryan Rebello pour son aide dans la rédaction de la légende de la figure 1.

Correspondance : Adam Komorowski, adam.komorowski@medportal.ca 